\title{
MiR-222 in Cardiovascular Diseases: Physiology and Pathology
}

\author{
Shengguang Ding, Haitao Huang, Yiming Xu, Hao Zhu, and Chongjun Zhong \\ Department of Thoracic and Cardiovascular Surgery, The Second Affiliated Hospital of Nantong University, Nantong 226001, China \\ Correspondence should be addressed to Chongjun Zhong; zhongcjnt001@126.com
}

Received 6 October 2016; Revised 7 December 2016; Accepted 13 December 2016; Published 3 January 2017

Academic Editor: Xinli Li

Copyright (C) 2017 Shengguang Ding et al. This is an open access article distributed under the Creative Commons Attribution License, which permits unrestricted use, distribution, and reproduction in any medium, provided the original work is properly cited.

\begin{abstract}
MicroRNAs (miRNAs and miRs) are endogenous 19-22 nucleotide, small noncoding RNAs with highly conservative and tissue specific expression. They can negatively modulate target gene expressions through decreasing transcription or posttranscriptional inducing mRNA decay. Increasing evidence suggests that deregulated miRNAs play an important role in the genesis of cardiovascular diseases. Additionally, circulating miRNAs can be biomarkers for cardiovascular diseases. MiR-222 has been reported to play important roles in a variety of physiological and pathological processes in the heart. Here we reviewed the recent studies about the roles of miR-222 in cardiovascular diseases. MiR-222 may be a potential cardiovascular biomarker and a new therapeutic target in cardiovascular diseases.
\end{abstract}

\section{Introduction}

Cardiovascular disease is a predominant cause of morbidity and mortality in the world [1]. The number of patients suffering from cardiovascular disease is growing larger and larger. The major categories of cardiovascular disease include disease of the blood vessels and the myocardium. The contemporary view thinks that most cardiovascular diseases resulted from a complex dysregulation of genetics and environmental factors. Also there are many molecular components that participate in this process, including noncoding RNAs.

MicroRNAs (miRNAs and miRs) are endogenous 19-22 nucleotide, small noncoding RNAs with highly conservative and tissue specific expression. miRNAs can modulate mRNA levels through decreasing transcription or posttranscription induced mRNA decay [2]. Since the first discovery of miRNAs in 1993, they have been found in many species and could participate in various physiological and pathological processes [3-6]. So far, there are over 1000 miRNAs that have been identified, among which at least 200 miRNAs are consistently expressed in the cardiovascular system [7]. miRNAs can regulate cardiomyocytes hypertrophy, senescence, apoptosis, autophagy, and metabolism. Changes of miRNAs have been found to participate in the genesis of many diseases including cardiovascular diseases [8].

miR-222, firstly discovered in human umbilical vein endothelial cells (HUVECs), has been reported to play important roles in epithelial tumors evidenced by its frequently increased expressions in epithelial tumors [9]. Reduction of miR-222 could inhibit cell proliferation and induce mitochondrial-mediated apoptosis through directly targeting the p53 upregulated modulator of apoptosis (PUMA) in breast cancer [10]. Its function on proliferation has also been confirmed in glioblastomas, thyroid papillary cancer, breast cancer, pancreatic cancer, hepatocellular carcinoma, and lung cancer [11-15]. On the other hand, miR-222 can play tumorsuppressive roles through the downregulation of c-kit in erythroleukemia cells. Apart from its role in cancer progress, miR-222 has been found to participate in many physiological and pathological processes in the cardiovascular system (Table 1). Here we reviewed the recent studies about the roles of miR-222 in cardiovascular diseases. MiR-222 may be a potential cardiovascular biomarker and a new therapeutic target in cardiovascular diseases.

\section{MiR-222 Regulates Physiological Function}

The role of miR-222 in regulating physiological process is on a cutting edge of studies (Figure 1).

2.1. MiR-222 Regulates Physiological Function in Cardiomyocytes. Physical exercise can induce cardiac growth mainly via hypertrophy and renewal of cardiomyocytes [16]. Unlike 


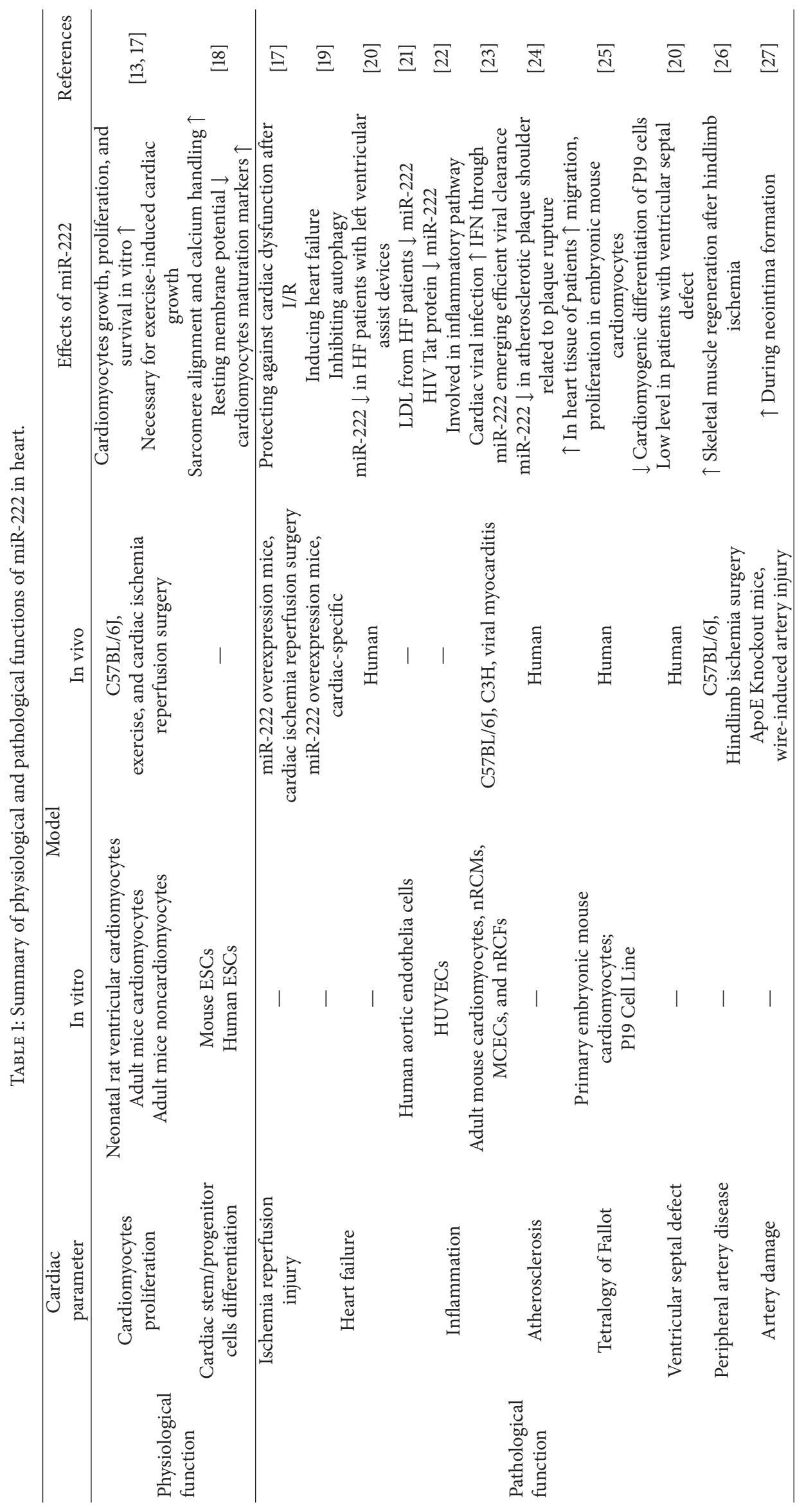




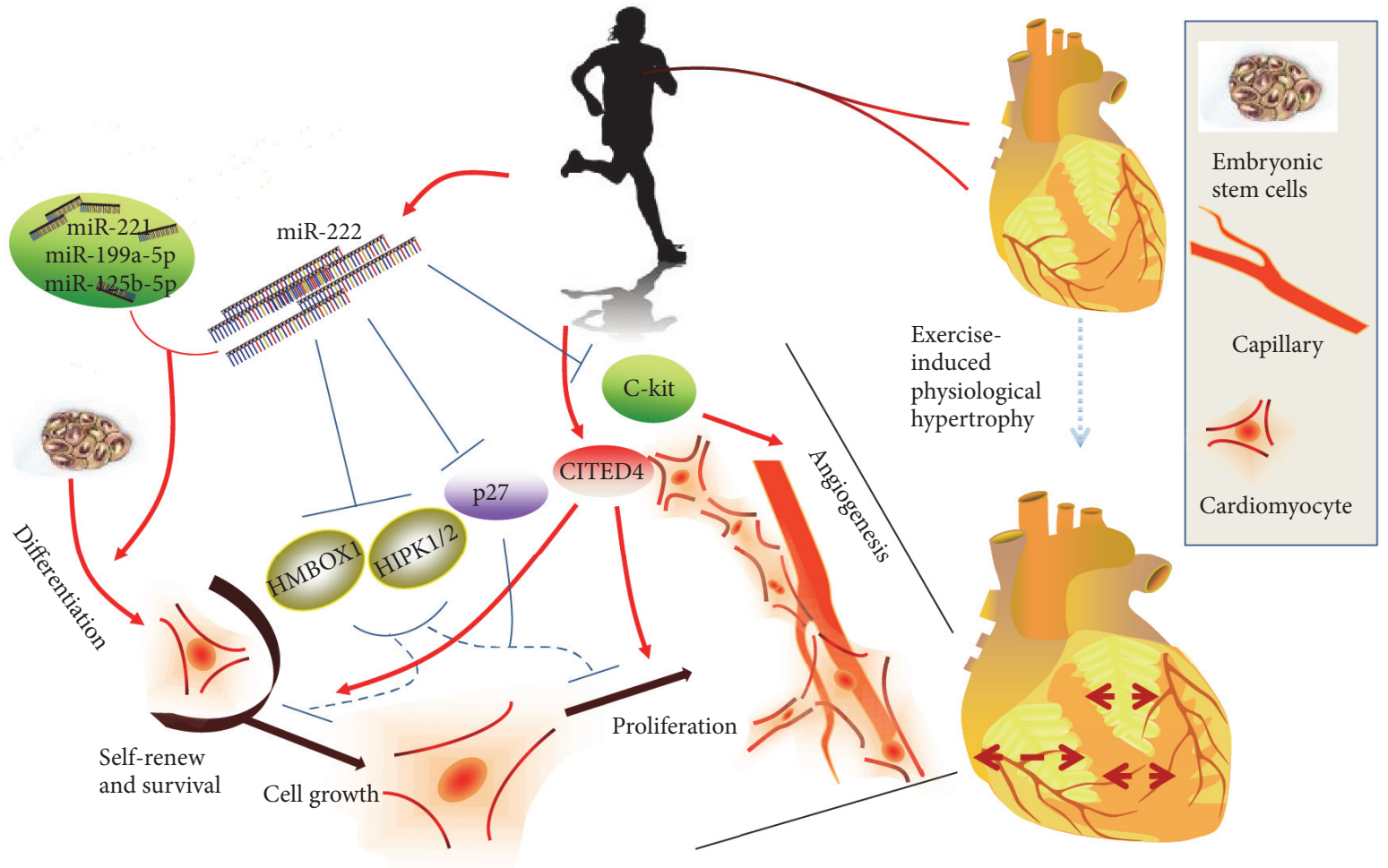

FIGURE 1: Multiple physiological functions of miR-222 (miR-222 has been found to participate in multiple physiological functions in cardiovascular system. In cardiac myocyte, miR-222 could promote cardiomyocytes growth, proliferation, and survival through directly targeting P27, HIPK-1, HIPK-2, and CITED-4 in traditional exercise pathway. In stem cell, miR-222 could promote CSCs transformation. In umbilical vein endothelial cells, miR-222 could exert angiogenesis function by targeting c-Kit).

pathological hypertrophy, which is related to myocardial structural disorder and cardiac dysfunction, physiological hypertrophy is characterized by normal cardiac structure and normal or improved cardiac function [28]. MiR-222 expression levels were found to be commonly increased in two distinct models of exercise, namely, voluntary wheel running and a ramp swimming exercise model as well as the exercise rehabilitation after heart failure in human. MiR-222 was able to promote cardiomyocytes hypertrophy, proliferation, and survival through directly targeting p27, HIPK-1, HIPK-2, and HMBOX1 [17].

2.2. MiR-222 Regulates Physiological Function in Cardiac Stem Cells. Heart has limited regenerative capacity, which might be based on cardiomyocyte division and cardiac stem and progenitor cell activation [29]. Cardiac stem cells (CSCs) are self-renewing, clonogenic, and multipotent, and they can differentiate to mature cardiomyocytes and improve the function and regeneration of the cardiovascular system [30]. CSCs can be activated by physical exercise training [18]. Interestingly, it has been found that the upregulation of miR-222 induced by coculturing human embryonic-stem cell-derived cardiomyocytes (m/hESC-CMs) with endothelial cells could increase and promote CSCs transformation to cardiomyocyte [18].

\subsection{MiR-222 Regulates Physiological Function in Human} Umbilical Vein Endothelial Cells. Human umbilical vein endothelial cells (HUVECs) have unique ability to form capillary-like structures in response to some stimuli. MiR222 has been reported to exert angiogenesis function through modulating HUVECs angiogenic activity by targeting c-Kit $[31,32]$.

2.4. Sex-Specific Expression of miR-222. There are differences between men and women in cardiovascular diseases incidence, while studies show that males are more likely to suffer from heart attacks than females [33, 34]. MiR222 are encoded on the $\mathrm{X}$ chromosome in mouse, rat, human and have sex-specific expression. Studies have indicated that miR-222 was specifically decreased in mature female mouse hearts as compared with male mouse hearts $[31,35]$. 


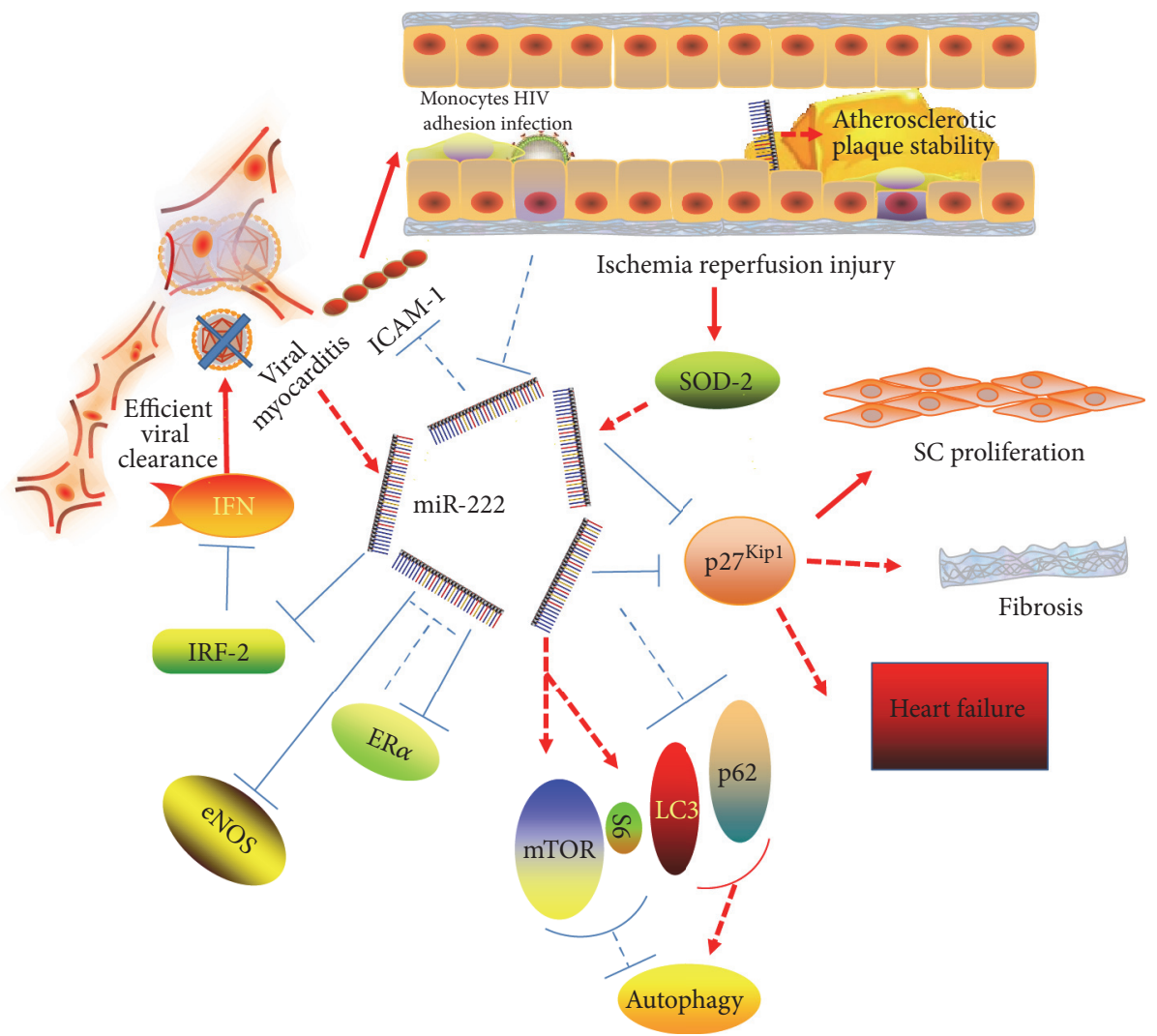

Figure 2: Multiple pathological functions of miR-222 (miR-222 has been found to participate in multiple pathological functions in cardiovascular system. In myocardium, miR-222 could (1) promote cardiomyocyte proliferation and reduce cardiomyocyte apoptosis through P27 after ischemic injury; (2) inhibit autophagy through mTOR; (3) regulate blood vessels remolding through c-Kit and eNOS; (4) regulate ICAM-1 and IRF-2 to inhibit inflammation. In blood vessels, miR-222 could (1) stable the plaque and suppress the inflammation and (2) inhibited the proliferation of vascular smooth muscle by targeting $\mathrm{p} 27)$.

\section{MiR-222 Regulates Pathological Function}

Unraveling the role of miR-222 in regulating cardiac pathological function may foster new therapeutic targets for cardiovascular diseases (Figure 2).

\subsection{MiR-222 Regulates Pathological Function in Myocardium}

3.1.1. Cardiac Ischemia Reperfusion Injury. Myocardial ischemic reperfusion is a complex process involving numerous mechanisms including reactive oxygen species (ROS) overload, inflammation and calcium overload, energy metabolism dysfunction, and mitochondrial permeability transition pore (mPTP) opening [36-38]. MiR-222 has been reported to be able to protect against cardiac dysfunction after ischemic injury. MiR-222 can promote cardiomyocyte proliferation and reduce cardiomyocyte apoptosis through P27. In addition, miR-222 overexpression mice have well-preserved cardiac function and reduced cardiac fibrosis when subjected to cardiac ischemia reperfusion [17].

3.1.2. Heart Failure. Heart failure is the terminal outcome of the majority of cardiovascular diseases, and it seriously reduces the quality of life. A significant inhibition of autophagy in Tg-miR-222 mice after heart failure was observed, which was through mTOR, a negative regulator of autophagy [19]. Inhibition of autophagy induced by miR222 may cause accumulation of protein and organelles injury, even the impairment of cardiac function. Angiogenesis has been proposed as a promising therapy for ischemia heart disease and heart failure. miR-221/222 family seemed to inhibit angiogenesis [21]. MiR-222 was significantly decreased in endothelial cells (ECs) when cultured for $24 \mathrm{~h}$ with HDL from chronic heart failure $(\mathrm{CHF})$ patients compared to healthy control. The downregulation of miR-222 may be a compensatory mechanism of ECs to counteract cardiovascular adverse events [39].

3.1.3. Viral Myocarditis. Cardiac inflammation is an important cause of dilated cardiomyopathy and heart failure. In young healthy adults, it can cause sudden death. Viral myocarditis is one of cardiac inflammation diseases. MiR222 has been reported to be able to orchestrate the antiviral and anti-inflammatory response through downregulation of IRF-2 [23]. Inhibition of miR-222 would increase the risk of cardiac injury. HIV-infected cardiomyopathies is another kind of inflammation diseases [22, 40]. MiR-222 can regulate cell adhesion molecules ICAM-1 translation directly or indirectly (through IFN- $\gamma$ ) to inhibit inflammation $[22,41]$. 
3.1.4. Congenital Heart Disease. Tetralogy of Fallot (TOF) is one of the most common congenital heart malformations in children [42]. miR-222 was found to display a high expression level in right ventricular outflow tract (RVOT) tissues compared with controls. Cardiac myocyte proliferation and differentiation is a key event in heart development. Further functional analysis showed that overexpression of miR-222 promoted cell proliferation and regulated cell differentiation by inhibiting the expression of the cardiomyocyte marker genes during the cardiomyogenic differentiation [25]. In another congenital heart disease, ventricular septal defect, the decreased expression of miR-222 also indicated its important role in heart development [20].

\subsection{MiR-222 Regulates Pathological Function in Blood Vessels}

3.2.1. Atherosclerosis. During the genesis of atherosclerosis, there are various molecules and cellular components that can make atherosclerotic plaque vulnerable and even rupture [43]. Many studies show that miRNAs also participate in this process [44]. MiR-222 derived from ECs may play its protective role by blocking intraplaque neovascularization and suppressing the inflammatory activation of ECs, without enhancing the proliferation of ECs $[45,46]$.

3.2.2. Peripheral Arterial Disease. Smooth muscle cells (SMCs) constitute the medial layer of arteries and regulate the vascular tone via their contractile apparatus [27]. MiR-222 was reported to take part in the development of neointima and promotes neointima formation after vascular injury by enhancing the proliferation of SMCs. Furthermore, in the peripheral artery disease (PAD) caused by atherosclerosis or inflammation of the peripheral arteries, studies have showed that miR-222 also inhibited the proliferation of vascular smooth muscle cell by targeting p27 [45] to stable the plaque [24] and promoted skeletal muscle regeneration after ischemia. Besides that, under the administration of superoxide dismutase-2 (SOD-2), miR-222 plays its protective role against peripheral artery disease by regulating p57 expression [26] but not P27.

\section{Conclusions}

In conclusion, miR-222 controls many cardiac physiological functions and its deregulation has been implicated in many cardiovascular diseases. Targeting miR-222 might be a promising therapeutic target for cardiovascular diseases.

\section{Competing Interests}

The authors declare there is no conflict of interests.

\section{Authors' Contributions}

Shengguang Ding, Haitao Huang, and Yiming Xu contributed equally to this work.

\section{References}

[1] A. Moran, D. Gu, D. Zhao et al., "Future cardiovascular disease in China markov model and risk factor scenario projections from the coronary heart disease policy model-China," Circulation, vol. 3, no. 3, pp. 243-252, 2010.

[2] S. Vimalraj and N. Selvamurugan, "MicroRNAs expression and their regulatory networks during mesenchymal stem cells differentiation toward osteoblasts," International Journal of Biological Macromolecules, vol. 66, pp. 194-202, 2014.

[3] R. C. Lee, R. L. Feinbaum, and V. Ambros, "The C. elegans heterochronic gene lin-4 encodes small RNAs with antisense complementarity to lin-14," Cell, vol. 75, no. 5, pp. 843-854, 1993.

[4] G. Wu, "Plant microRNAs and development," Journal of Genetics and Genomics, vol. 40, no. 5, pp. 217-230, 2013.

[5] J. Qian, Z. Zhang, J. Liang et al., "The full-length transcripts and promoter analysis of intergenic microRNAs in Drosophila melanogaster," Genomics, vol. 97, no. 5, pp. 294-303, 2011.

[6] H. Quach, L. B. Barreiro, G. Laval et al., "Signatures of purifying and local positive selection in human miRNAs," American Journal of Human Genetics, vol. 84, no. 3, pp. 316-327, 2009.

[7] I. Bentwich, A. Avniel, Y. Karov et al., "Identification of hundreds of conserved and nonconserved human microRNAs," Nature Genetics, vol. 37, no. 7, pp. 766-770, 2005.

[8] E. Van Rooij, L. B. Sutherland, N. Liu et al., "A signature pattern of stress-responsive microRNAs that can evoke cardiac hypertrophy and heart failure," Proceedings of the National Academy of Sciences of the United States of America, vol. 103, no. 48, pp. 18255-18260, 2006.

[9] S. Volinia, G. A. Calin, C.-G. Liu et al., "A microRNA expression signature of human solid tumors defines cancer gene targets," Proceedings of the National Academy of Sciences of the United States of America, vol. 103, no. 7, pp. 2257-2261, 2006.

[10] C. Zhang, J. Zhang, A. Zhang et al., "PUMA is a novel target of miR-221/222 in human epithelial cancers," International Journal of Oncology, vol. 37, no. 6, pp. 1621-1626, 2010.

[11] C. Le Sage, R. Nagel, D. A. Egan et al., "Regulation of the $\mathrm{p} 27^{\text {Kip } 1}$ tumor suppressor by miR-221 and miR-222 promotes cancer cell proliferation," EMBO Journal, vol. 26, no. 15, pp. 3699-3708, 2007.

[12] R. Visone, L. Russo, P. Pallante et al., "MicroRNAs (miR)-221 and miR-222, both overexpressed in human thyroid papillary carcinomas, regulate p27Kip1 protein levels and cell cycle," Endocrine-Related Cancer, vol. 14, no. 3, pp. 791-798, 2007.

[13] T. E. Miller, K. Ghoshal, B. Ramaswamy et al., "MicroRNA221/222 confers tamoxifen resistance in breast cancer by targeting p27Kip1," Journal of Biological Chemistry, vol. 283, no. 44, pp. 29897-29903, 2008.

[14] X. Fu, Q. Wang, J. Chen et al., "Clinical significance of miR221 and its inverse correlation with p27 Kip1 in hepatocellular carcinoma," Molecular Biology Reports, vol. 38, no. 5, pp. 30293035, 2011.

[15] M. Garofalo, C. Quintavalle, G. Di Leva et al., "MicroRNA signatures of TRAIL resistance in human non-small cell lung cancer," Oncogene, vol. 27, no. 27, pp. 3845-3855, 2008.

[16] L. Tao, Y. Bei, H. Zhang, J. Xiao, and X. Li, "Exercise for the heart: signaling pathways," Oncotarget, vol. 6, no. 25, pp. 2077320784, 2015.

[17] X. Liu, J. Xiao, H. Zhu et al., "MiR-222 is necessary for exerciseinduced cardiac growth and protects against pathological cardiac remodeling," Cell Metabolism, vol. 21, no. 4, pp. 584-595, 2015. 
[18] D. S. Lee, J.-H. Chen, D. J. Lundy et al., "Defined microRNAs induce aspects of maturation in mouse and human embryonicstem-cell-derived cardiomyocytes," Cell Reports, vol. 12, no. 12, pp. 1960-1967, 2015.

[19] M. Su, Z. Chen, C. Wang et al., "Cardiac-specific overexpression of miR-222 induces heart failure and inhibits autophagy in mice," Cellular Physiology and Biochemistry, vol. 39, no. 4, pp. 1503-1511, 2016.

[20] D. Li, L. Ji, L. Liu et al., "Characterization of circulating microRNA expression in patients with a ventricular septal defect," PLoS ONE, vol. 9, no. 8, Article ID e106318, 2014.

[21] S. Riedel, S. Radzanowski, T. S. Bowen et al., "Exercise training improves high-density lipoprotein-mediated transcription of proangiogenic microRNA in endothelial cells," European Journal of Preventive Cardiology, vol. 22, no. 7, pp. 899-903, 2015.

[22] M. Duan, H. Yao, G. Hu, X. Chen, A. K. Lund, and S. Buch, "HIV tat induces expression of ICAM-1 in HUVECs: implications for miR-221/-222 in HIV-associated cardiomyopathy," PLoS ONE, vol. 8, no. 3, Article ID e60170, 2013.

[23] M. Corsten, W. Heggermont, A.-P. Papageorgiou et al., "The microRNA-221/-222 cluster balances the antiviral and inflammatory response in viral myocarditis," European Heart Journal, vol. 36, no. 42, pp. 2909-2919, 2015.

[24] H. A. Bazan, S. A. Hatfield, C. B. O’Malley, A. J. Brooks, D. Lightell, and T. C. Woods, "Acute loss of miR-221 and miR222 in the atherosclerotic plaque shoulder accompanies plaque rupture," Stroke, vol. 46, no. 11, pp. 3285-3287, 2015.

[25] J. Zhang, J.-J. Chang, F. Xu et al., "MicroRNA deregulation in right ventricular outflow tract myocardium in nonsyndromic tetralogy of fallot," Canadian Journal of Cardiology, vol. 29, no. 12, pp. 1695-1703, 2013.

[26] G. Togliatto, A. Trombetta, P. Dentelli et al., "Unacylated ghrelin promotes skeletal muscle regeneration following hindlimb ischemia via SOD-2-mediated miR-221/222 expression," Journal of the American Heart Association, vol. 2, no. 6, p. e000376, 2013.

[27] F. Zahedi, M. Nazari-Jahantigh, Z. Zhou et al., "Dicer generates a regulatory microRNA network in smooth muscle cells that limits neointima formation during vascular repair," Cellular and Molecular Life Sciences, 2016.

[28] E. D. Abel and T. Doenst, "Mitochondrial adaptations to physiological vs. pathological cardiac hypertrophy," Cardiovascular Research, vol. 90, no. 2, pp. 234-242, 2011.

[29] O. Bergmann, R. D. Bhardwaj, S. Bernard et al., "Evidence for cardiomyocyte renewal in humans," Science, vol. 324, no. 5923, pp. 98-102, 2009.

[30] A. A. Kocher, B. Schlechta, A. Gasparovicova, E. Wolner, N. Bonaros, and G. Laufer, "Stem cells and cardiac regeneration," Transplant International, vol. 20, no. 9, pp. 731-746, 2007.

[31] P. Dentelli, A. Rosso, F. Orso, C. Olgasi, D. Taverna, and M. F. Brizzi, "MicroRNA-222 controls neovascularization by regulating signal transducer and activator of transcription $5 \mathrm{~A}$ expression," Arteriosclerosis, Thrombosis, and Vascular Biology, vol. 30, no. 8, pp. 1562-1568, 2010.

[32] D. de Gonzalo-Calvo, A. Cenarro, F. Civeira, and V. LlorenteCortes, "microRNA expression profile in human coronary smooth muscle cell-derived microparticles is a source of biomarkers," Clinica e Investigacion en Arteriosclerosis, vol. 28, pp. 167-177, 2016.

[33] B. C. Bernardo, X.-M. Gao, C. E. Winbanks et al., “Therapeutic inhibition of the miR-34 family attenuates pathological cardiac remodeling and improves heart function," Proceedings of the
National Academy of Sciences of the United States of America, vol. 109, no. 43, pp. 17615-17620, 2012.

[34] M. Tanasescu, M. F. Leitzmann, E. B. Rimm, W. C. Willett, M. J. Stampfer, and F. B. Hu, "Exercise type and intensity in relation to coronary heart disease in men," Journal of the American Medical Association, vol. 288, no. 16, pp. 1994-2000, 2002.

[35] A. M. Evangelista, A. M. Deschamps, D. Liu, N. Raghavachari, and E. Murphy, "miR-222 contributes to sex-dimorphic cardiac eNOS expression via ets-1," Physiological Genomics, vol. 45, no. 12, pp. 493-498, 2013.

[36] Y. Zhang, B. Wang, X. Fu et al., "Exogenous NAD+ administration significantly protects against myocardial ischemia/reperfusion injury in rat model," American Journal of Translational Research, vol. 8, no. 8, pp. 3342-3350, 2016.

[37] F. Bagheri, V. Khori, A. M. Alizadeh, S. Khalighfard, S. Khodayari, and H. Khodayari, "Reactive oxygen species-mediated cardiac-reperfusion injury: mechanisms and therapies," Life Sciences, vol. 165, pp. 43-55, 2016.

[38] X. Huang, L. Zuo, Y. Lv et al., "Asiatic acid attenuates myocardial ischemia/reperfusion injury via Akt/GSK-3 $\beta /$ HIF- $1 \alpha$ signaling in rat H9c2 cardiomyocytes," Molecules, vol. 21, no. 9, article 1248, 2016.

[39] F. P. Hans, M. Moser, C. Bode, and S. Grundmann, "MicroRNA regulation of angiogenesis and arteriogenesis," Trends in Cardiovascular Medicine, vol. 20, no. 8, pp. 253-262, 2010.

[40] G. Barbaro and S. E. Lipshultz, "Pathogenesis of HIV-associated cardiomyopathy," Annals of the New York Academy of Sciences, vol. 946, pp. 57-81, 2001.

[41] G. Hu, A.-Y. Gong, J. Liu, R. Zhou, C. Deng, and X.-M. Chen, "miR-221 suppresses ICAM-1 translation and regulates interferon- $\gamma$-induced ICAM-1 expression in human cholangiocytes," American Journal of Physiology-Gastrointestinal and Liver Physiology, vol. 298, no. 4, pp. G542-G550, 2010.

[42] J. P. Starr, "Tetralogy of fallot: yesterday and today," World Journal of Surgery, vol. 34, no. 4, pp. 658-668, 2010.

[43] E. Hergenreider, S. Heydt, K. Tréguer et al., "Atheroprotective communication between endothelial cells and smooth muscle cells through miRNAs," Nature Cell Biology, vol. 14, no. 3, pp. 249-256, 2012.

[44] F. Jansen, X. Yang, S. Proebsting et al., "MicroRNA expression in circulating microvesicles predicts cardiovascular events in patients with coronary artery disease," Journal of the American Heart Association, vol. 3, no. 6, Article ID 001249, 2014.

[45] X. Liu, Y. Cheng, S. Zhang, Y. Lin, J. Yang, and C. Zhang, "A necessary role of miR-221 and miR-222 in vascular smooth muscle cell proliferation and neointimal hyperplasia," Circulation Research, vol. 104, no. 4, pp. 476-487, 2009.

[46] X. Liu, Y. Cheng, J. Yang, L. Xu, and C. Zhang, "Cell-specific effects of miR-221/222 in vessels: molecular mechanism and therapeutic application," Journal of Molecular and Cellular Cardiology, vol. 52, no. 1, pp. 245-255, 2012. 


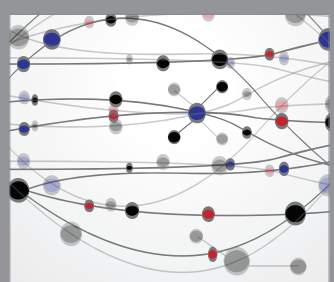

The Scientific World Journal
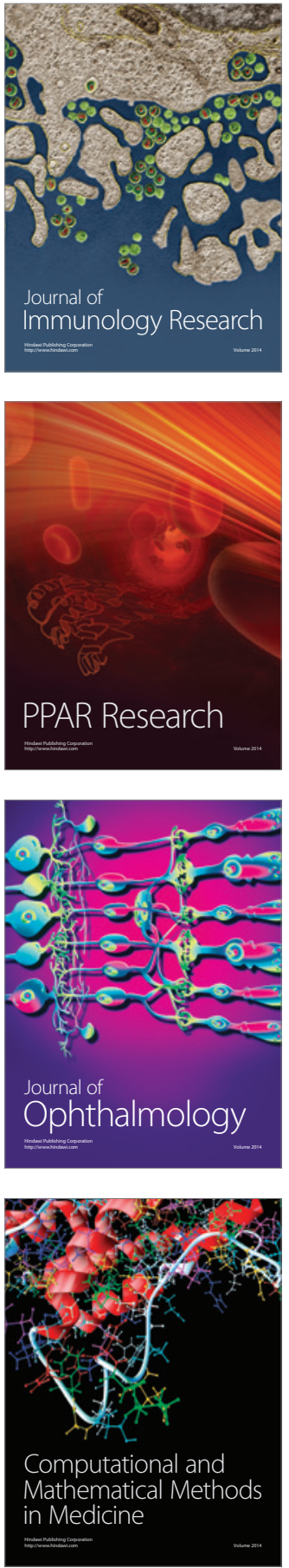

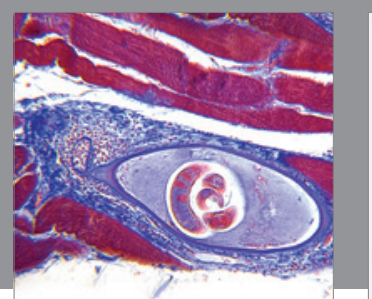

Gastroenterology Research and Practice
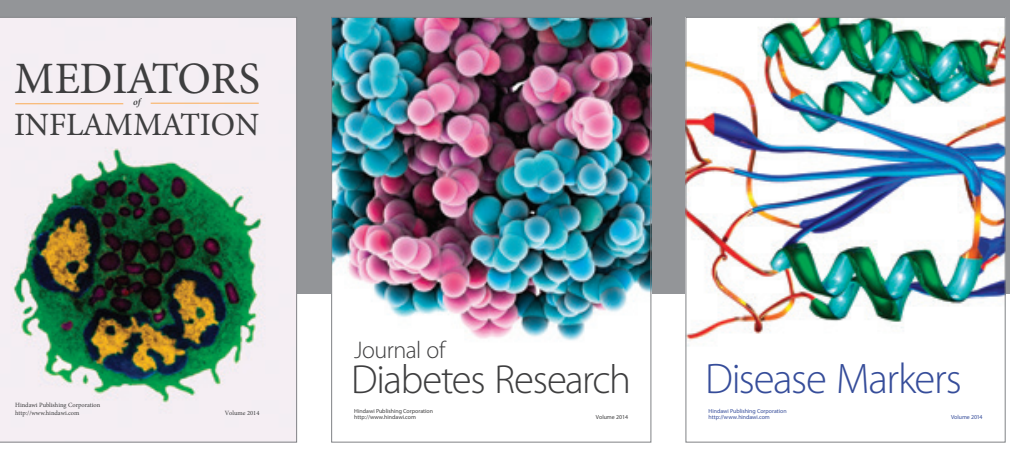

Disease Markers

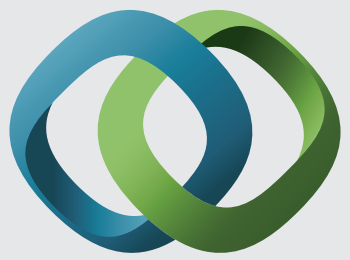

\section{Hindawi}

Submit your manuscripts at

https://www.hindawi.com
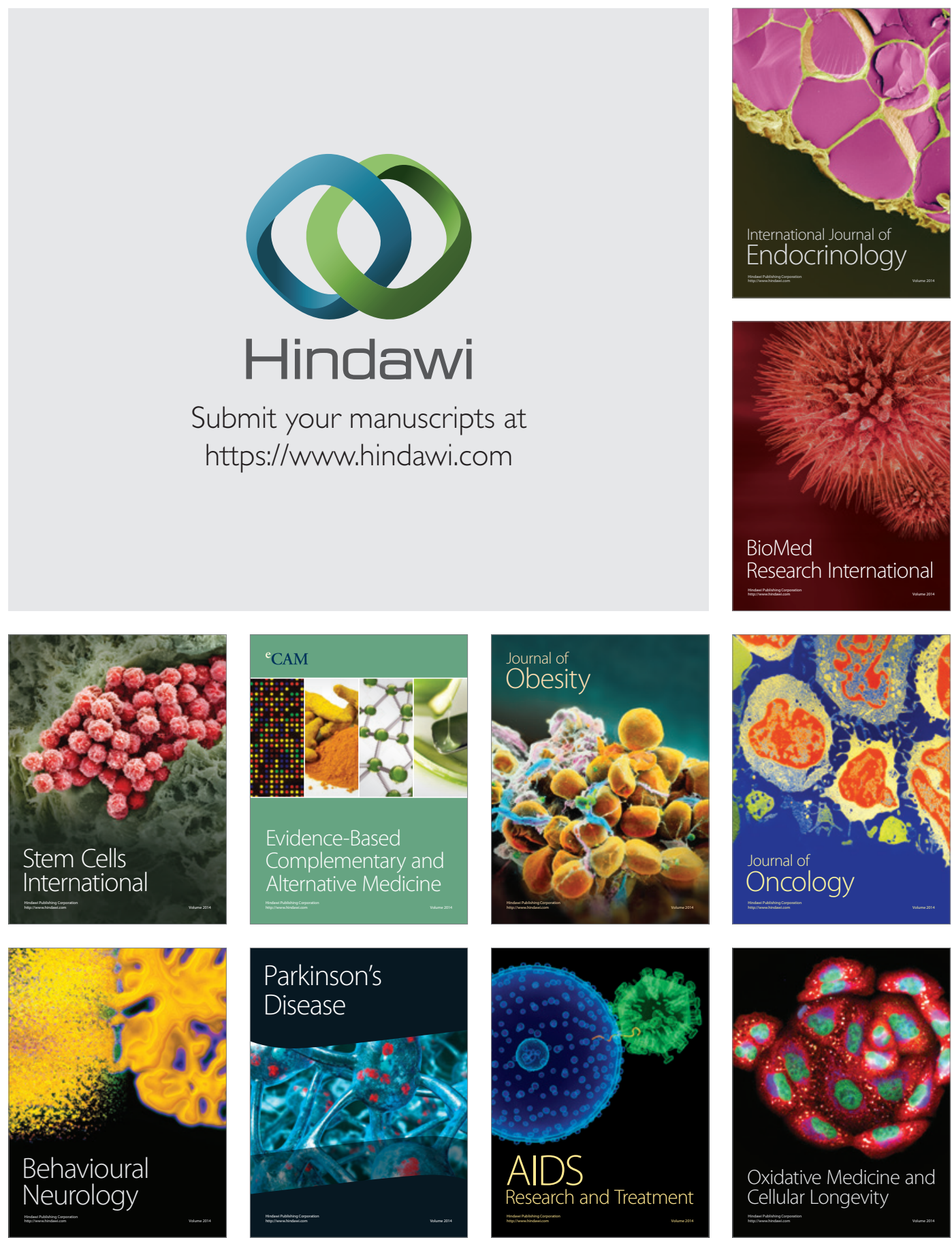